\title{
Performance of an Improved TEM SDD Detector
}

\author{
Hendrik O. Colijn ${ }^{1}$, Fan Yang ${ }^{1}$, D. B. Williams ${ }^{1}$, Alan Sandborg ${ }^{2}$, David W. McComb ${ }^{1}$ \\ 1. Center for Microscopy and Analysis, The Ohio State University, Columbus, OH, USA \\ 2. EDAX, Inc., Mahwah, NJ, USA
}

Silicon drift detectors (SDD) have become the standard X-ray detector on SEMs and are increasingly common on TEMs as well [1]. Count rates and low energy sensitivity for the SDDs are significantly improved over the older $\mathrm{Si}(\mathrm{Li})$ detectors [2]. The in-situ SDD systems have multiple detectors and hence have a significant improvement in solid angle over the standard $\mathrm{Si}(\mathrm{Li})$ detectors.

External SDD systems can also replace $\mathrm{Si}(\mathrm{Li})$ detectors on existing microscopes and offer many of the advantages of the in-situ SDD systems. Since the SDDs do not operate at liquid nitrogen temperatures, the insulation gaps in the nosepiece/collimator assemblies can be reduced or eliminated. Also, the detector window and the associated support frame are no longer used. This means that the detecting area can be moved closer to the sample improving the solid angle for signal collection. In addition, the fact that the detector operates at $-20 \mathrm{C}$ to $-30 \mathrm{C}$ instead of cryogenic temperatures means that ice buildup on the detector is no longer an issue. One limitation to increasing the solid angle is that the detector must fit through the port in the sample stage area. While detectors are being made with larger and larger active areas, if they can't fit through the microscope port they are useless.

We report here on an improved design of a $30 \mathrm{~mm}^{2}$ SDD mounted on an FEI Tecnai F20 with an X-Twin objective lens. This SDD replaces an earlier version of a $30 \mathrm{~mm}^{2}$ SDD. Like the initial design the detector is retractable to protect it from high X-ray fluxes and back-scattered electrons. Figure 1 illustrates the improvement in count rate with the new design relative to the original. These measurements were done with the standard FEI double-tilt sample holder. At a sample tilt angle of $+15^{\circ}$ there is a nearly $50 \%$ improvement in count rate for the $\mathrm{NiK} \alpha$ peak in the $\mathrm{NiO}$ test sample. Figure 2 illustrates the light element sensitivity of the detector where the counts are normalized to the silicon intensity. It should be noted that there is a drop in the FeL and $\mathrm{O} K$ peak intensities at $0^{\circ}$ sample tilt. This appears to be due to a difference in the absorption paths for the two detectors.

With the detector inserted so close to the sample, the collimator doesn't significantly limit the field of view. Moving the detector in or out has little or no effect as to whether the collimator occludes the view of the sample. Extraneous fluorescence contributions due to brehmsstrahlung, however, will be seen by the detector. It follows that good hard X-ray and pole-piece shielding is especially important when using these SDDs. The Tecnai F20 TEMs can be operated with the adjustable $\mathrm{C} 1$ aperture as the beam limiting aperture. As shown in Figure 3, this reduces the hole-count spectrum by over a factor of 10 [3].

\section{References:}

[1] H.S. von Harrach et al, Microscopy \& Microanalysis 15, S2, 208 (2009)

[2] P.G. Kotula, J.R. Michael, Microscopy \& Microanalysis, (2013), 978.

[3] The authors acknowledge support from The Ohio State University and the Ohio Third Frontier

Research Scholar program. 

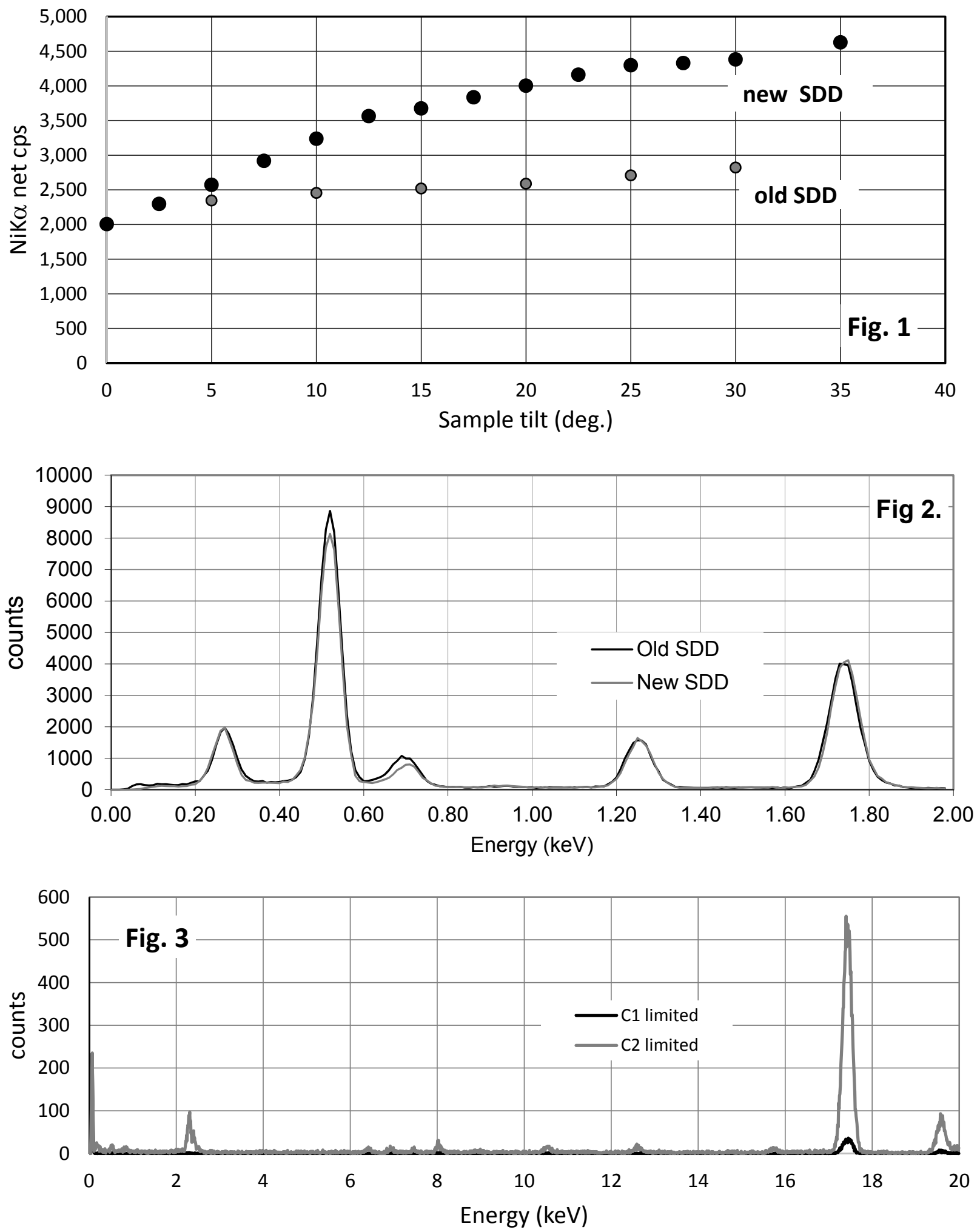

Figure 1. NiK $\alpha$ count rate for a $65 \mathrm{~nm} \mathrm{NiO}$ film vs sample tilt angle scaled to $1 \mathrm{nA}$.

Figure 2. Low energy from SRM 2063 at $0^{\circ}$ sample tilt.

Figure 3. Hole count comparison with different beam limiting apertures 